\title{
Abstracts from the Poster Session of the 2017 American Massage Therapy Association National Convention
}

\section{MASSAGE FOR BREAST CANCER SURVIVORS: PRESENTING EVIDENCE AND IDENTIFYING OPPORTUNITIES}

Emiliana Bomfim, BScN, MSc, PhD (cand), Kalyani Premkumar, $\mathrm{MD}, \mathrm{PhD}$

Introduction: Although massage therapy (MT) has been providing positive results, the quantity and quality of available preliminary evidences is not sufficient to build trustworthy clinical guidelines, especially to address long-term treatment-related symptoms (TRS) in breast cancer survivors. Certainly, this lack of evidence prevents us from bridging the gap between basic research and clinical practice, and at the same time, requires an extensive compilation and discussion of the evidence already published in order to unveil the possibilities of scientific and clinical exploration.

Objective: The objective of this work is to present evidence on massage therapy as a TRS reduction strategy and its immune response-stimulating effects in breast cancer survivors, as well as present the current gaps and limitations of research in this arena.

Methods: From a standpoint of translational research, a scooping review was performed. Five databases were used as data sources: LILACS, EMBASE, CINAHL, PubMed, and the Cochrane Library. Inclusion criteria were full-text articles available in English, Spanish or Portuguese and published in the last 20 years (1981-2016).

Results: From the preselected articles, 12 clinical trials were retained for critical appraisal on methodological quality. Overall, the literature highlights many advantages in the use of CAM by breast cancer patients, suggesting its value in long-term TRS management. Overall, the evidence gathered by this review shows that many MT types show a positive effect on the levels of different biomarkers such as IgA levels, $\alpha$-amylase, salivary cortisol, salivary flow rate, serum cortisol, serotonin, NK cells, lymphocytes, urinary catecholamines, Th1 molecules (IL-1, IFN- $\gamma$ ), Th2 (IL-4, IL-10), peripheral blood mononuclear cell, hemodynamic effects, and increase of dopamine and serotonin levels. The concept of cancer survivor care has received greater attention due to the increasing number of cancer survivors living well beyond their initial diagnosis. Despite the increased survival rate, there is a continuing need for research to assess the effects of Complementary and Alternative Medicine (CAM) on patient's quality of life (QoL) and TRS.

Conclusions: Most of MT that were tested in the studies consistently improved the quality of life of breast cancer patients. Overall, the use of MT seems to be an effective, safe, and noninvasive approach in the management of several TRS for breast cancer survivors.

\section{EVALUATING IMPACT OF SWEDISH MASSAGE ON SH GIRDLE AND C4-T1 NERVE ROOTS POSTINJECTION THERAPY}

\section{Kierra Douglas}

Introduction: Thoracic Outlet Syndrome is a condition where the nerves, arteries, or veins are compressed by structures that make up the thoracic outlet. There is no treatment protocol for working with patients receiving anesthetic injection treatments for pain management along with massage therapy.

Objectives: Objectives of this case study are to evaluate the impact of Travell trigger point massage, joint oscillations, and Swedish massage on the shoulder girdle inclusive of C4-T1 nerve roots, postlocal anesthetic injection treatments.

Methods: The patient, a 52-year old female, diagnosed with thoracic outlet syndrome bilaterally with increased symptoms on left side. She received 8 treatments over five weeks, 2 treatments a week with a one-week break. Treatments included techniques such as Travell trigger point release, joint oscillations, and Swedish massage. Pretreatment and posttreatment assessments of Myotome and Dermatome testing of the upper torso, and selected resisted muscle tests of the neck and arm, were used to evaluate resulting impact.

Results: In the three metrics measuring pain, there was a significant decrease observed by the patient over the five weeks of treatment. Muscle functioning improved over the duration of the study.

Conclusion: Travell trigger point massage, joint oscillations, and Swedish massage on the shoulder girdle inclusive of C4-T1 nerve roots, postlocal anesthetic injection treatments, has proven to decrease patient pain and increase patient's ability to participate in activities of daily living. Although the findings were positive for the researchers' hypothesis, further 
research will be needed to prove the significance and effectiveness of the combination of therapeutic massage and anesthetic injection therapy.

\section{INTEGRATED KNOWLEDGE TRANSLATION IN MASSAGE THERAPY RESEARCH: RESULTS OF STAKEHOLDER CONSULTATION}

Donelda M. Gowan, RMT, MSc, PhD (cand), Anne Leis, $\mathrm{PhD}$

Introduction: Massage therapy (MT) research is a nascent field, and the input of MT experts including regulators, professional association leaders, practitioners, and educators in the conduct of research is needed. The research-informed development of a patient safety culture, an important topic in health care, is critical to the professionalization and maturation of a profession. Yet, there is a dearth of information about MT patient safety issues, informed by consultation with massage therapists.

Purpose: The aim of this research was to apply an integrated knowledge translation approach to the conduct of a MT research project about patient safety.

Methods: Approval of the study was granted by the University of Saskatchewan Behavioral Research Ethics Board. Following a well-established knowledge translation process, consultation with approximately 40 stakeholders within the Canadian massage therapy community informed a doctoral research project. Consultation activities occurred in Western and Eastern Canada and included meetings, an educational conference, a focus group, and a workshop presentation to solicit stakeholder input and feedback.

Results: The main results of the integrative knowledge translation project included a provincial leadership motion to support a patient safety-related research project, engagement with practitioners aided in exploring the research questions, and analysis of focus group participants responses revealed themes that were developed into a model of patient safety. Stakeholder consultation revealed perceived value in the study with recommendations to disseminate the results widely to policy-makers including regulators and practitioners, with specific direction to use professional magazines for education and sharing of the new knowledge.

Conclusions: The use of an integrated knowledge translation framework served to break new ground in the Canadian MT community by building relationships of trust between the researchers, regulators, and practitioners. Examining the professional discourse about patient safety among the Canadian MT community revealed the underlying need for standardization in title, licensing, and practice.

\section{BODY AWARENESS PROGRAM FOR MENTAL HEALTH CONSUMERS}

Lori Ruth Federman Kalvert, MA, LMT, RYT, SME, Greta Winograd, PhD

Introduction: The National Institute of Mental Health reports that, in 2015 , approximately $17.9 \%$ of Americans have some diagnosable mental illness. At least $4 \%$ have serious mental illness, and are likely to have accompanying physical comorbidities and a life expectancy 25 years less than the rest of the US population. Helping individuals find ways to improve coping and reduce stress may help improve health outcomes.

Purpose: A qualitative analysis which seeks to describe the experiences and perceptions of participants with severe mental illness who participated in a 16-week massage and mental wellness program.

Methods: A three-tiered 16-week program focused on massage and mental wellness was implemented at a mental health clubhouse. Tiers included: 1) focusing on the self with self-massage through acupressure and kinesthetic ball work, 2) reaching out to the other through partner ball massage, and 3) expanding exposure to wellness community by receiving professional massage and taking a field trip to a wellness/ yoga center. All instruction was experienced within the framework of a yoga class. The program was delivered by an LMT with five years of experience who worked closely with the staff at the clubhouse to facilitate continuity of care. Seven participants, 2 men and 5 women between the ages of 25 and 75, all except one being diagnosed with a variety of severe and persistent mental illnesses, were interviewed and audio-recorded about their experience with the program. The recordings were transcribed and through an iterative process, two researchers used inductive methods to independently identify themes and subthemes within interviews, which were verified by a third researcher. This study and evaluation was approved by Fountain House Institutional Review Board, IRB \# 00001400, and the SUNY New Paltz Institutional Review Board.

Results: The themes identified were program success, self-awareness, and positive experiences. Program success was determined as the participants indicated that they experienced an increase in body awareness and coping skills, as well as stress reduction mirroring the goals of the program. Participants' increased self-awareness is indicated by two subthemes: 1) increase in social skills requiring trust and vulnerability with others, and 2) experiencing self-insight and self-acceptance with regard to trust and vulnerability within oneself. Finally, the positive experiences theme is supported by the descriptions of all interviewees and their expressions of trust and vulnerability in their praise/gratitude towards instructor. 
Conclusion: A program focused on touch and massage was successfully introduced to mental health consumers over time in a clubhouse setting. Results suggest that, from a participant perspective, the program helped them to gain skills and improve awareness, and was a positive experience. Access to massage and self-massage appeared to foster individual and social benefits to the participants in the group. Although this was a small study with only seven participants being interviewed, it appears that the participants may have gained more resilient boundaries with oneself and others through an experience of trust and vulnerability. The humanistic, nonpathologizing design of this massage program may be a key to this metatheme, and further research into similar programs is warranted.

\section{IMPACT OF REFLEXOLOGY ON NUMBER OF CIGARETTES SMOKED BY A 56-YEAR-OLD FEMALE: A CASE REPORT}

\section{E. LoResa Robertson, LMT, CST}

Introduction: Cigarette smoking leads to $\$ 280$ billion in health care costs and lost productivity, and over 393,000 deaths worldwide on an annual basis. Smoking is a way for people to cope with stress. Reflexology can also be used for stress reduction.

Objective: To identify the short-term impact of reflexology on number of cigarettes smoked by a chronic smoker.

Case Presentation and Intervention: A 56-yearold computer analyst was offered 15-minute reflexology treatments twice a day during her regular breaks for 20 consecutive workdays. She presented as a high dependency smoker $(20+$ cigarettes daily $)$ with neck and shoulder pain.

Interpretation: The client chose reflexology treatment in lieu of smoking on all 20 days. She decreased her cigarette use from $20+$ to $8-10$ cigarettes per day and stated that her urge to smoke decreased. Her neck and shoulder Numeric Pain Rating Scale (NPRS) scores reduced from $9 / 10$ to $2 / 10$ over the 20 days, and her Fagerstrom Test for Cigarette Dependency (FTCD) scores reduced from $5 / 9$ to $3 / 9$. She also noted that she was sleeping longer at night, felt more rested and energized, had an improved appetite, and was more relaxed overall. Treatment was discontinued after the 20 workdays and the client resumed smoking $20+$ cigarettes per day.

Implications: Reflexology may be beneficial as an intervention for smoking cessation. There are several possible explanations for this treatment benefit including a reduction in anxiety, depression, or physical pain. Even though reflexology provided positive outcomes in this case report, more research is needed to determine the most effective treatment approach, dosage, and duration of treatment for chronic smokers. Studies are also needed regarding physiological mechanisms of action of reflexology for smoking cessation.

\section{THE USE OF MASSAGE TO INCREASE RANGE OF MOTION AND DECREASE PAIN IN A BRAZILIAN JIU-JITSU ATHLETE: A CASE REPORT}

\author{
Alta Schwab, LMT, NST
}

Introduction: Brazilian Jiu-Jitsu is a modern combat martial art that utilizes joint locks to force an opponent into submission. It is a high intensity combat sport that places unique stresses on the body leading to atypical injuries and muscular imbalance, a possible predictor of injury. Maximum joint ROM is necessary for optimal performance, and athletes with a history of low back pain have significant reduction in hip ROM. Massage has been linked to improved range of motion (ROM) and pain reduction. Maximum ROM is essential for optimum athletic performance and pain can inhibit ROM and function of joints.

Participant: A 40-year-old Caucasian male Jiu Jitsu black belt presenting with restricted ROM in the right shoulder and both hips, as well as left posterior gluteal pain. The patient was diagnosed by his orthopedist with herniated L4-5 disc and no other notable health issues. Informed consent was acquired prior to beginning treatment.

Purpose: To discover if the utilization of massage can increase ROM and decrease pain in a BJJ athlete training at an intense level.

Intervention: The practitioner is a 25 -year-old female within 3 months of the end of her third semester of a three-semester, 18-month program at the Center for Neurosomatic Studies (CNS). Biweekly targeted, deep-tissue massage treatments were performed for five weeks. The results were tracked using the Bournemouth Musculoskeletal Pain Scale (BQ-MSK), the Western Ontario Shoulder Instability Index (WOSI), active range of motion measurements measured with a goniometer, and Patients Global Impression of Change Scale.

Results: Posterior gluteal pain improved (64\% improvement/raw change score, pre- 11 to post- 7). There was improvement in the function of the shoulder based on the WOSI score pre-427 to post-13. ROM of the shoulder improved, goniometer changes measured the following increases in motion: Flexion: pre- $139^{\circ}$ to post- $167^{\circ}$; extension: pre- $55^{\circ}$ to post$57^{\circ}$; abduction: pre- $113^{\circ}$ to post- $163^{\circ}$; and external rotation pre- $53^{\circ}$ to post- $83^{\circ}$. The practitioner noted visual improvement of the elbow and the hip range of motion. The PGIC score changed from pre- 1 to post5 , indicating moderate improvement. The patient also 
claimed to have experienced more range of motion, stating that his back and hip is looser and his SI joint pain was eliminated.

Conclusion: Specific, directed massage techniques appear to have improved ROM and decreased pain in one BJJ athlete. An extenuating factor for pain reduction included an abnormally intense and frequent training regimen for an upcoming tournament. Further research is needed on the use of massage in a BJJ athlete in order to ascertain if increasing ROM and decreasing pain may be effective in increasing athletic performance.

\section{NEW ZEALAND ELITE RUGBY UNION PLAYERS' UTILIZATION OF MASSAGE THERAPY}

\section{Donna Smith, PhD; Sarah Carter, BTSM}

Introduction: New Zealand's use of massage therapy within the sporting environment has increased extensively in the past decade and now plays an important role within the country's national sport of rugby union, particularly for elite-level players. While massage therapy is utilized by professional rugby players, little is known about the players' perceived benefits of massage therapy, how they utilize massage therapy, and their perceptions of massage therapy and massage therapists.

Objective: The purpose of this research was to highlight the key areas of New Zealand elite rugby union players' utilization of massage therapy.

Methods: This pilot study adopted a qualitative approach using semi-structured interviews (telephone $\mathrm{n}=1$; face-to-face $\mathrm{n}=2$ ) of approximately 15 minutes duration in May 2015. Participants were three New Zealand professional rugby union players who had played rugby union for the New Zealand All Blacks at some point in their careers. The interviews were transcribed verbatim with pseudonyms used for the participant's names. The transcripts were thematically analyzed. Ethical approval for this research project was granted by the Southern Institute of Technology Research Ethics Committee.

Results: The primary reasons for use of massage therapy were: 'recovery after training of a game', 'relaxation', and 'injury rehabilitation'. Frequency of Use varied from rarely during the off season to twice weekly during the season. Massage therapy was perceived as being: 'enjoyable', 'important', and 'consistency is key'. The essential characteristics of massage therapists reported were: knowledge of anatomy, professionalism, confidence, firm pressure, and approachable.

Conclusions: The small sample size is a limitation of this study. However, this pilot study suggests that elite rugby union players in New Zealand hold massage therapy in high regard. Massage therapy is seen as an important part of sports medicine, and the participants believe it has assisted with their longevity as professional rugby union players.

\section{DO MALES HAVE ADVANTAGES OR DISADVANTAGES IN THE MASSAGE THERAPY INDUSTRY? SOUTHLAND MALE PRACTITIONERS' PERSPECTIVES}

\author{
Jo Smith, PhD, Philip O'Kane, BTSM
}

Introduction: Gender inequality, discrimination, and sexist perceptions have been observed historically within many occupations, and several professions have a disproportionate representation of one gender. Whether females are more suited to jobs involving sensitivity, nurturance, and beauty, and whether males are more suited to skill, strength, and labor-related work has been speculated historically. Massage therapy is a profession with a workforce of around $80 \%$ females, raising questions such as: Are females more suited to the massage profession and, if so, why? Massage is an intimate form of therapy requiring the removal of clothes and trust in one's practitioner. Public perceptions linking sex to massage therapy, male social stigma, and sexual identity of clients may all be variables that influence how clients choose their massage practitioner. With massage therapy comes a complex dynamic of gender relations and little corresponding specific research.

Aim: The purpose of this pilot study was to examine the advantages and disadvantages of being a male massage therapist, and to gain insight into individual strategies to negotiate gender-related issues.

Methods: Following ethics approval, face-to-face individual semi-structured interviews ( $\mathrm{n}=5, \mathrm{NZ}$ male massage therapists) were conducted in May 2014.

Results: This research identified several advantages and disadvantages for male massage therapists; however, the significance and implications upon their practice appeared minimal. A general strength advantage was agreed to by all participants, but this did not imply superior massage application or greater industry success. Any physical ascendancy that males may have seemed to be proportionately offset by a different, possibly smaller client pool due to gender/ sexuality comfort issues. Additionally, males may have to deal with collective gender reputation damage due to current or historical male practitioner transgressions and unfavorable residual public perceptions from the past. Strategic recommendations from the participants to counter male gender disadvantages included: sound professional boundaries, good communication skills, immaculate draping, and pride in personal presentation.

Conclusion: The small number of participants limits any generalizablity from this study. However, 
overall this research concluded that male massage therapists do have minor advantages and disadvantages in the massage therapy industry, but practitioner success was not dictated by gender - rather by individual skill, professionalism, and reputation development.

\section{INVERCARGILL-BASED GENERAL PRACTITIONERS: PERCEPTIONS OF MASSAGE THERAPY}

\author{
Jo Smith, PhD, Rebecca Whitaker, BTSM
}

Introduction: There has been a significant increase in the use of massage therapy over the last ten years. Referral rates from medical practitioners have risen annually, as more scientific research is conducted on the efficacy of massage therapy and the support for integration of massage therapy into conventional health care increases. However there is limited information regarding New Zealand General Practitioners' (GPs) perceptions and practices of referral to massage therapy.

Aim: The purpose of this NZ-based pilot study was to explore GPs views on massage therapy in relation to conventional health care, and examine attitudes and barriers to referral to massage therapy.

Methods: Face to face, 15-minute semi-structured individual interviews $(n=4)$ with Southland, New Zealand-based GPs. Transcripts were thematically analyzed. Ethics approval was granted by the Southern Institute of Technology Research Ethics Committee.

Results: Three significant barriers to referral were highlighted: 'a time factor', 'a general lack of knowledge on massage therapy as a whole', and 'a lack of understanding of who massage therapists are and what they do'. Adequate interprofessional communication between massage therapists (MTs) and GPs was identified as lacking on a three-tier level including: from individual MTs to GPs, at an individual practice level, and from the professional massage therapy industry at large. 'General health and wellbeing' and 'symptoms that related to musculoskeletal dysfunction' were the conditions GPs would consider referring patients to massage therapy. Massage therapy was: 'an option to consider', and there was a need for further 'exploration of how to work together' with the conventional health care industry.

Implications: While the findings from a small number of participants are not representative, they do generate discussion. It appears that ongoing massage therapy research, with disseminated and accessible results, is greatly needed to inform GPs and assist them with their decision-making process, to encourage appropriate massage therapy referral. There is also a need for improved communication and information flow with regard to massage therapy education and massage therapist credentials. GPs were open and generally positive about the use of massage therapy. They lacked resources and information regarding massage therapy, therapists' qualifications, and skill set, which potentially hindered referral practice.

\section{RESTLESS LEG SYNDROME AND MASSAGE THERAPY: A CASE REPORT}

\section{Samantha Zabel, MA, BA, AAS}

Introduction: Approximately 15\% of Americans experience disruptive symptoms associated with Restless Legs Syndrome (RLS), including difficulty sleeping, fatigue, and a decreased quality of life. Little research exists on the effectiveness of massage therapy in people with RLS, but it is a commonly used method of self-care.

Purpose: This case report examined the effects of regular massage therapy on a 46-year-old female with symptoms consistent with RLS who, as a result of symptoms, experienced daily fatigue that affects her quality of life. Goals included improving quality of sleep by reducing RLS symptoms and reducing fatigue.

Methods: The interventions were adapted from a previously published case report about a client with RLS. The client, who was self-diagnosed with RLS, kept a daily sleep log, tracking aspects of sleep such as number of, and reasons for, disturbances (including number and intensity of RLS symptoms), caffeine and alcohol intake, and time taken to fall asleep. The client also completed the Pittsburgh Sleep Quality Inventory (PSQI) before and after the study. Massage therapy was given once a week for four weeks, in 45- to 60-minute sessions. Techniques included Western massage (effleurage, petrissage, compressions), contract-relax, and stretching, addressing the whole body, but focusing on the lower body.

Results: The client's PSQI score improved slightly from a score of 8 at the beginning of the study to 7 at the end. Her subjective perception of fatigue improved from "fairly bad" to "fairly good", and she reported a decrease in number and intensity of RLS symptoms.

Conclusions: Massage therapy may help decrease symptoms of RLS, resulting in improved sleep quality. Although the results of this and the previously published case report are encouraging, further research is required to adequately explore the effects of massage therapy on RLS and sleep quality. 